Original Research Paper

\title{
Nattokinase Activity Guided Natto Fermentation Process Optimization
}

\author{
${ }^{1,2}$ Xiaojie Ren, ${ }^{1}$ Chao Huang, ${ }^{1}$ Xin Shan, ${ }^{1}$ Heng Ban, ${ }^{1}$ Baoyue Liu, ${ }^{3} Y u$ Du, ${ }^{1}$ Yuanda Song and ${ }^{1,3,4}$ Xinhe Zhao \\ ${ }^{1}$ Colin Ratledge Center for Microbial Lipids, School of Agriculture Engineering and Food Science, \\ Shandong University of Technology, Zibo, China \\ ${ }^{2}$ Baolingbao Biology Co., Ltd., Dezhou, China \\ ${ }^{3}$ Chongqing Academy of Science and Technology, Chongqing, China \\ ${ }^{4}$ State Key Laboratory of Microbiology, Shandong University, Qingdao, China
}

Article history

Received: 16-09-2021

Revised: 09-11-2021

Accepted: 12-11-2021

Corresponding Author:

Xinhe Zhao

School of Agriculture

Engineering and Food Science,

Colin Ratledge Center for

Microbial Lipids, China

E-mail: zhaoxinhe2018@163.com

\begin{abstract}
The natto kinase in natto has obvious thrombolytic effect, has the advantages of low cost, small side effects, long half-life and can be taken orally, so it has attracted much attention. At present, the research on natto kinase in our country mainly focuses on the selection of natto fermentation strains, the optimization of gene expression conditions and the exploration of thrombolytic mechanism. However, most natto products show that the number of viable bacteria is not high. Natto Kinase activity is low and no breakthrough has been made so far. For this reason, this topic has launched a study on how to improve the activity of natto kinase. This topic optimizes the traditional natto fermentation process and obtains the best fermentation parameters on the basis of enhancing the activity of natto kinase. The experiment first compared and selected two different fermentation strains and determined that Bacillus natto was the dominant fermentation strain. Considering that the water content of soybeans can indirectly affect the activity of natto kinase, the experiment explored the effects of soaking and steaming beans on the water content of soybeans and determined that soaking beans is an influencing factor for the fermentation process. During the fermentation process, soaking time, fermentation temperature, fermentation time and inoculation amount of superior species, were designed to explore the optimal fermentation process that make the highest nano kinase activity. The TAME method is used to accurately detect the activity of natto kinase in the samples after fermentation and the physical properties of the natto before and after fermentation are measured with a physical property test set. The data obtained can quantify the subjective sensory evaluation. Finally, the designexport 11.0 software was used to analyze and optimize the optimal fermentation process parameters with a soaking time of $15 \mathrm{~h}$, a fermentation temperature of $37^{\circ} \mathrm{C}$, a fermentation time of $30 \mathrm{~h}$ and an inoculum of $6 \mathrm{~mL}$ and the natto kinase activity under the combined conditions was measured to be $3 \mu \cdot \mathrm{mL}^{-1}$.
\end{abstract}

Keywords: Natto, Natto Kinase Activity, TAME Method, Response Surface

\section{Introduction}

Natto is made from soybean as raw material and fermented by Natto bacteria (Bacillus subtilis natto). During the fermentation of soybeans, various enzymes such as cellulase, amylase, glucoamylase, lysozyme, Superoxide Dismutase (SDS) (Tamura and Takenaka, 1999), etc. are produced, which can break down the protein in natto into small molecules. Peptides or amino acids are more easily absorbed and utilized by the body (Iwai et al., 2002). In addition, natto contains a kind of natto kinase (Natto kinase, NK), it belongs to fibrinolytic enzymes and can decompose throm boxin formed in blood vessels, dissolve thrombus strongly and prevent cerebral infarction and myocardial infarction (Wang et al., 2021); Compared with traditional urokinase drugs such as streptokinase, it has the advantages of rapid thrombus dissolution, long half-life and fewer side effects. In addition to these valuable advantages, natto kinase also has other uses, including the treatment of hypertension, alzheimer's disease and vitreoretinal diseases, etc. (Jensen et al., 2016; Takagaki et al., 2020). The attractive market prospects make natto kinase become the focus of more people and the large-scale production of natto kinase has also become a research hotspot for many companies. 
Natto kinase has many advantages, such as wide source of fermentation materials, short production cycle, high yield, low price and easy to be extracted and purified, therefore, it has a broad prospect (Ju et al., 2019; Junfeng et al., 2020; Vianney et al., 2019). Compared with liquid fermentation, solid fermentation has low cost, high enzyme activity, rich enzyme system, simple operation of equipment, easy to large-scale production and high yield (Xiaodan et al., 2021). Therefore, this process is widely used in domestic and foreign food fermentation industrial production.

In recent years, Japan has taken the lead in research on Natto and related products. There are natto products enriched with Bacillus subtilis and related products enriched and purified by natto kinase. Although there are a lot of relevant studies in China, the overall level still needs to be improved, mainly manifested as low live count of Bacillus subtilis and low natto kinase activity and no breakthrough has been made so far (Zexin et al., 2017). In this study, based on the traditional fermentation technology and the previous research results, through the optimization of soybean fermentation process, aiming at the high yield of natto kinase, the solid-state fermentation conditions were optimized, in order to obtain the industrial fermentation process conditions for the production of high activity natto kinase and provide support for the development of related products.

\section{Materials and Methods}

\section{Fermentation Bacteria Preparation}

The strains of the fermented natto are Bacillus subtilis natto and Lactobacillus plantarum and the two are streaked in LB solid medium and MRS solid medium respectively. Static culture in the thermostat at $37^{\circ} \mathrm{C}$, after a single clear colon appear on the plate, pick it into a test tube which filled with 4 to $6 \mathrm{~mL}$ of liquid medium and rejuvenating in the shaker of $37^{\circ} \mathrm{C}, 200 \mathrm{rpm}$. The bacterial culture in the test tube was inoculated into $100 \mathrm{~mL}$ liquid medium at $2 \%$ when the alignment period $(\mathrm{OD}=0.8)$ is reached, it is then inoculated according to certain proportion into the cryogenic soybean.

\section{Natto Fermentation Process}

Take and weigh $50 \mathrm{~g}$ soybeans and clean them. Put them into a stainless-steel basin with a lid and soak in distilled water with 3 times the amount of soya beans for a period of time. Pour water, cover the basin with two layers of gauze, cover it and cook it in an autoclaving pot at $121^{\circ} \mathrm{C}$ for $20 \mathrm{~min}$. After cooling, add a certain proportion of bacteria liquid. After mixing the soybean and bacteria, spread it on the bottom of the basin and put it into a constant temperature incubator for fermentation for a certain time. Finally, it was transferred to $4^{\circ} \mathrm{C}$ refrigerator and cooked for $24 \mathrm{~h}$ to obtain natto products (Shih et al., 2013).

\section{Effect of Soaking and Steaming Beans on Soybean Water Content}

It has been known that the anthropic kinase activity is affected by the water content of soybeans. In the fermentation process, the factors that affect the water content of soybeans were soaking and the steam process. In the experiment, $50 \mathrm{~g}$ soybean was soaked for $6 \mathrm{~h}$ and $22 \mathrm{~h}$ respectively. After soaking, the water was drained and weighed and its weight was recorded. Then four $50 \mathrm{~g}$ soybeans were weighed and soaked for $6 \mathrm{~h}$. One of them was boiled at $121^{\circ} \mathrm{C}$ for $20 \mathrm{~min}$ and the other was boiled at $121^{\circ} \mathrm{C}$ for $40 \mathrm{~min}$. After cooling, they were weighed and their weights were recorded. Soak the remaining two portions for $22 \mathrm{~h}$, cooking time and temperature as above, weigh and record the grams after cooling (Yongjun et al., 2010).

\section{Optimizing Process Parameters of Natto Fermentation using Response Surface Methodology}

In this study, the soybean soaking time, fermentation temperature, fermentation time and the inoculation amount of bacteria were used as influencing factors to optimize the fermentation conditions of natto for the activity of natto kinase. Attention should be paid to the inoculation, in order to ensure the uniformity of the experiment, the total volume of inoculation are $10 \mathrm{~mL}$ and the bacterial liquid less than $10 \mathrm{~mL}$ should be filled with distilled water. For example: When the inoculation volume is $2 \mathrm{~mL}$, an additional $8 \mathrm{~mL}$ of distilled water needs to be added and the two are evenly mixed before pouring into the soybeans. Using the fourfactor five-level quadratic rotation orthogonal combination response surface (CangXue et al., 2019) experiment to design random conditional combination parameters (Table 1), a total of 30 sets of experiments and finally after fermentation according to the combined conditions, the enzyme activity is determined (Hailin, 2014). The soybean used in the experiments all came from the same bag and production. A brief chart was provided to show the experiment design as below Fig. 1 .

Table 1: Experimental factors and horizontal design

\begin{tabular}{lllll}
\hline No. & Soybean soaking time/h & Fermentation temperature $/{ }^{\circ} \mathrm{C}$ & Fermentation time/h & Inoculum amount $/ \mathrm{ml}$ \\
\hline 1 & 6 & 31 & 18 & 2 \\
2 & 10 & 34 & 23 & 4 \\
3 & 14 & 37 & 28 & 6 \\
4 & 18 & 40 & 33 & 8 \\
5 & 22 & 43 & 38 & 10 \\
\hline
\end{tabular}




\section{Determination of Physical Properties of Soybean}

The TMS-2000 physical property test set was used to determine the physical properties of soybeans before and after fermentation and the TPA method (Test parameters: Probe $\mathrm{P} 75$ head $(\Phi 75 \mathrm{~mm}$ pressure plate), test mode selection TPA, speed before test $5 \mathrm{~mm} / \mathrm{s}$, test speed $1 \mathrm{~mm} / \mathrm{s}$, after test speed $5 \mathrm{~mm} / \mathrm{s}$, pause $5.0 \mathrm{~s}$ between two compressions, sample compression deformation 90.0\%) was used to determine each group before and after fermentation (Pengyu et al., 2021). From each group, 10 to 13 samples of soybeans were selected for measurement and the average was taken. According to the characteristic curve of TPA texture, the parameters of TPA test are defined as follows: Hardness represents the force required for the sample to reach a certain deformation under the set conditions and the unit is g. Adhesiveness reflects the nature of soybean adhesion to the palate, teeth, tongue and other contact surfaces during chewing. The negative sign of the data indicates that the direction of the force applied to the probe is downward, regardless of the magnitude and the unit is $\mathrm{g} \cdot \mathrm{s}$. Springiness indicates the degree to which the sample can recover after the first compression; cohesiveness indicates that the sample resists damage and is tightly connected during the chewing process to keep it intact. It reflects the inherent nature of the sample. Springiness refers to the degree to which the sample can recover after the first compression. Cohesiveness refers to the properties of the properties of the sample resisting damage and tightly connecting to keep it intact during the chewing process, which reflects the internal cohesiveness of the sample. Chewiness is used to describe the work that a solid food needs to be chewed to be swallowed. It comprehensively reflects the continuous resistance of the sample of chewing. It is the product of hardness, cohesion and elasticity and the unit is $\mathrm{g}$. Resilience represents the ratio of the elastic energy released by the sample of the first compression cycle to the energy consumption of the probe during compression and is one of the parameters that characterize the elasticity of the sample.

\section{Extraction and Determination of Natto Kinase}

Take a number of natto that has been cooked after fermentation and grind it in a mortar until no solid particles are visible. Take a certain number of grams and put it in a beaker and add 6 to 7 times the mass of natto in $0.9 \%$ sterile saline solution. Disperse and mix in a homogenizing emulsifier. Pour the homogeneous solution into a centrifuge tube, freeze and centrifuge for $10 \mathrm{mins}$ at $4^{\circ} \mathrm{C}, 10,000 \mathrm{rpm}$ and the supernatant obtained is the crude natto kinase enzyme solution. The determination of natto kinase adopts the TAME method (Yinxin et al., 2006). That is, natto kinase decomposes TAME and the product methanol undergoes a series of color reactions to form a blue-violet complex and the absorbance of the complex is proportional to the activity of natto kinase. The specific operation is as follows: Take several test tubes with plugs, 2 blank tubes and the rest are sample tubes and each set of samples is done twice in parallel. Add $0.1 \mathrm{~mL}$ of TAME $\left(0.1 \mathrm{~mol} \cdot \mathrm{L}^{-1}\right), 0.1 \mathrm{~mL}$ of PBS solution, $0.1 \mathrm{~mL}$ of sample solution to the sample tube and replace the sample solution to the blank tube with $0.1 \mathrm{~mL}$ PBS. Put them into a water bath at $37^{\circ} \mathrm{C}$ for $30 \mathrm{~min}$. After taking it out, immediately cool about in an ice bath, while adding $0.2 \mathrm{~mL}$ of $15 \%$ trichloroacetic acid solution and shaking well to terminate the enzyme reaction. Then add $0.1 \mathrm{~mL}$ of $2 \%$ potassium permanganate, shake for 1 2 min to fully react, then add $0.1 \mathrm{~mL}$ of $10 \%$ anhydrous sodium sulfite to reduce the incompletely reacted potassium permanganate and finally add $4 \mathrm{~mL}$ of the prepared chromotopic acid solution, shake to mix well, put them in a boiling water bath for color reaction for $25 \mathrm{~min}$, take it out and cool down in an ice bath, measure the absorbance at $574 \mathrm{~nm}$ and convert it to the concentration of methanol through the methanol standard curve. Natto kinase activity is expressed in the number of decomposed TAME units. The number of TAME units is calculated according to the following formula.

TAME $\left(\mathrm{u} \cdot \mathrm{mL}^{-1}\right)=($ Methanol micromolecule number $\times$ sample dilution multiple)/(sample volume $\times$ time $)$.

\section{Results and Discussion}

\section{Selection of Fermentation Strains}

The experimental Lactobacillus plantarum and Bacillus subtilis natto were applied respectively to ferment $50 \mathrm{~g}$ soybeans under the same fermentation conditions. The activity of natto kinase was measured by TAME method and each strain was performed twice in parallel. The results are shown in Fig. 2. Among them, natto fermented with Lactobacillus plantarum has natto kinase activities of 0.63 and $0.57 \mu \cdot \mathrm{mL}-1$, respectively. The enzyme activity of natto fermented with Bacillus subtilis natto is as high as 2.01 and $2.1 \mu \cdot \mathrm{mL}-1$. It can be seen that the activity of natto kinase obtained from the fermentation of Bacillus subtilis natto is higher, which is more than twice as high as the activity of natto kinase obtained after fermentation with Lactobacillus plantarum. In order to achieve the purpose of improving the activity of natto kinase, the experiment selected Bacillus subtilis natto as the fermentation strain to continue optimization.

\section{The Effect of Soaking and Steaming Beans on the Moisture Content of Soybeans}

Considering that the water content of soybeans can indirectly affect the activity of natto kinase, the experiment explored the effects of soaking and steaming soybeans on the water content of soybeans and compared the weight difference of soybean before and 
after treatment. The results are shown in Fig. 3. The moisture content of soybeans soaked for $6 \mathrm{~h}$ is far less than that of soybeans soaked for $22 \mathrm{~h}$, which proves that the time of soaking soybeans has a significant impact on the moisture content of soybeans. After soaking the beans for $6 \mathrm{~h}$, the soybeans were steamed for 20 and $40 \mathrm{~min}$ and it was found that the quality difference before and after steaming the beans was only $1 \sim 2 \mathrm{~g}$. Moreover, the beans are soaked for $22 \mathrm{~h}$ and then steamed and the results obtained are the same as above, so it can be obtained that the steamed beans have no major influence on the moisture content of the soybeans. As the water content of soybeans significantly affects the activity of natto kinase, soaking soybeans is selected as the influencing factor to further to optimize the fermentation process parameters of natto.

\section{Determination of Optimum Fermentation Parameters}

During the fermentation process, the soybean soaking time (moisture content), fermentation temperature, fermentation time and the inoculation amount of dominant fermentation strains were changed and 30 sets of different factor combination experiments were designed using RSM based on the Central Composite Design (RSM-CCD). The natto obtained by fermentation was measured for natto kinase and the results are shown in Table 2.

The measured results were input into design-Expert 11.0 software and two results were obtained: The actual value and the predicted value of natto kinase activity. It can be seen from the measured actual value that under the combined conditions of $14 \mathrm{~h}-37^{\circ} \mathrm{C}-28 \mathrm{~h}-6 \mathrm{~mL}$, the activity of natto kinase measured after fermentation is the highest $3.37 \mu \cdot \mathrm{mL}^{-1}$; Under the combined conditions of $14 \mathrm{~h}-43^{\circ} \mathrm{C}-28 \mathrm{~h}-6 \mathrm{~mL}$, the measured natto kinase activity was the lowest, only $0.63 \mu / \mathrm{mL}$. It is preliminarily judged that the enzyme production of Bacillus subtilis natto is lower in an environment with a higher fermentation temperature, which may be because the optimal growth temperature of Bacillus subtilis natto is $37^{\circ} \mathrm{C}$. At a higher temperature of $43^{\circ} \mathrm{C}$, the survival rate is lower, so the measured enzyme activity is lower. From this preliminary analysis, it is concluded that the fermentation temperature has a significant effect on the enzyme production rate of natto fermentation.

Table 2: CCD experimental design and results

\begin{tabular}{|c|c|c|c|c|c|c|}
\hline No. & Soaking time/h & Temperature $/{ }^{\circ} \mathrm{C}$ & Time/h & D-inoculum/ml & NK actual value $\mu / \mathrm{ml}$ & NK predicted value $\mu / \mathrm{ml}$ \\
\hline$\overline{1}$ & 10 & 34 & 23 & 4 & 1.94 & 1.73 \\
\hline 2 & 10 & 34 & 23 & 8 & 1.89 & 1.86 \\
\hline 3 & 10 & 40 & 23 & 8 & 1.01 & 1.38 \\
\hline 4 & 14 & 37 & 28 & 6 & 2.78 & 2.99 \\
\hline 5 & 14 & 37 & 28 & 6 & 2.63 & 2.99 \\
\hline 6 & 10 & 34 & 33 & 8 & 1.46 & 1.69 \\
\hline 7 & 14 & 31 & 28 & 6 & 0.98 & 0.82 \\
\hline 8 & 14 & 37 & 38 & 6 & 2.82 & 2.35 \\
\hline 9 & 10 & 40 & 23 & 4 & 2.18 & 1.89 \\
\hline 10 & 14 & 37 & 28 & 6 & 3.37 & 2.99 \\
\hline 11 & 6 & 37 & 28 & 6 & 1.96 & 1.85 \\
\hline 12 & 18 & 40 & 23 & 8 & 1.84 & 1.63 \\
\hline 13 & 10 & 40 & 33 & 8 & 0.74 & 0.60 \\
\hline 14 & 14 & 37 & 28 & 10 & 1.75 & 1.44 \\
\hline 15 & 18 & 34 & 23 & 4 & 0.79 & 1.02 \\
\hline 16 & 14 & 37 & 28 & 6 & 3.23 & 2.99 \\
\hline 17 & 14 & 37 & 28 & 6 & 3.06 & 2.99 \\
\hline 18 & 18 & 34 & 23 & 8 & 1.49 & 1.40 \\
\hline 19 & 18 & 34 & 33 & 4 & 2.35 & 2.08 \\
\hline 20 & 18 & 40 & 23 & 4 & 2.03 & 1.90 \\
\hline 21 & 10 & 34 & 33 & 4 & 1.66 & 1.95 \\
\hline 22 & 18 & 40 & 33 & 8 & 1.37 & 1.68 \\
\hline 23 & 18 & 34 & 33 & 8 & 1.69 & 2.06 \\
\hline 24 & 14 & 43 & 28 & 6 & 0.63 & 0.61 \\
\hline 25 & 18 & 40 & 33 & 4 & 2.22 & 2.35 \\
\hline 26 & 14 & 37 & 28 & 2 & 1.86 & 1.98 \\
\hline 27 & 14 & 37 & 18 & 6 & 1.80 & 2.08 \\
\hline 28 & 14 & 37 & 28 & 6 & 2.86 & 2.99 \\
\hline 29 & 10 & 40 & 33 & 4 & 1.30 & 1.50 \\
\hline 30 & 22 & 37 & 28 & 6 & 2.30 & 2.23 \\
\hline
\end{tabular}



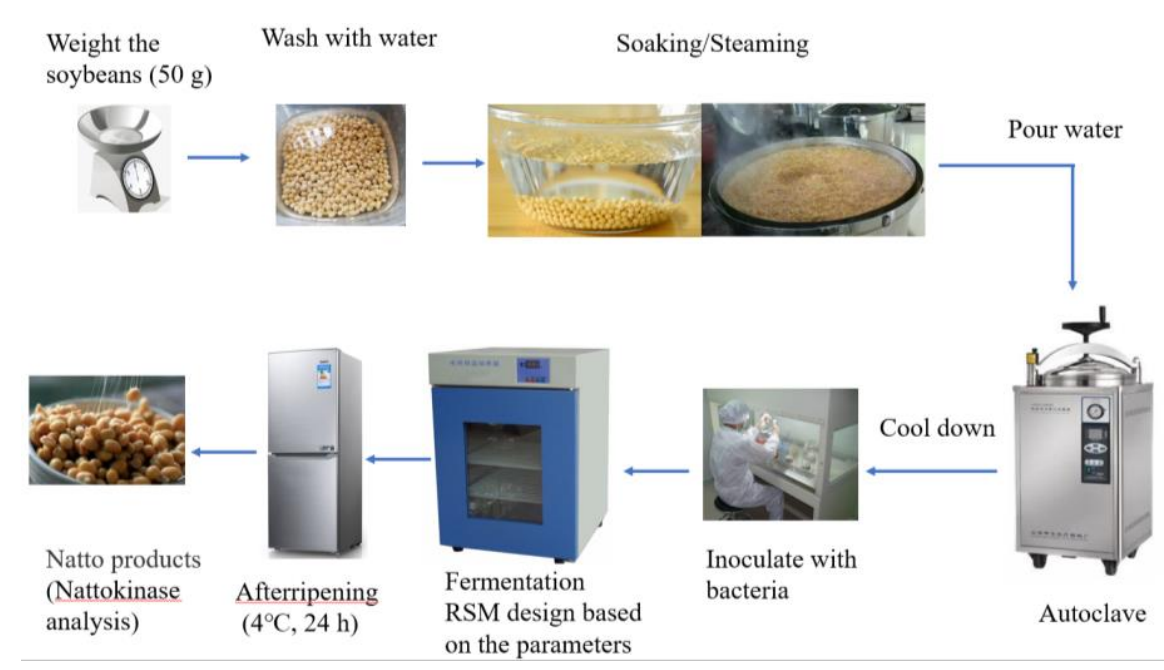

Fig. 1: Experimental design flow chart

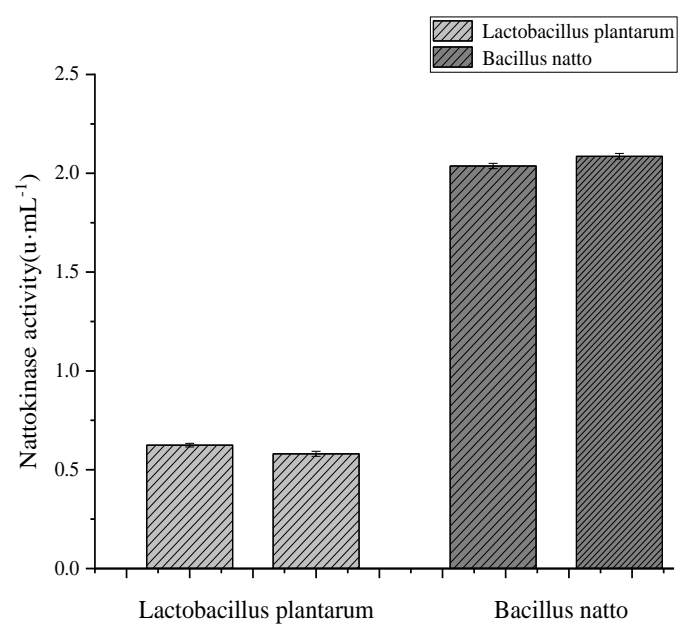

Fig. 2: Comparison of natto kinase activities fermented by different strains

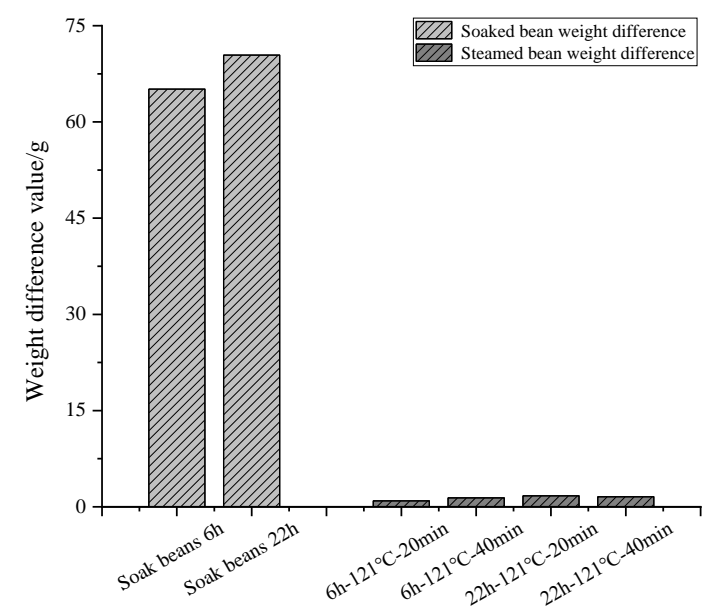

Fig. 3: Comparison of weight difference between soaked and steamed bean
Analyze the data according to Design-expert 11.0 software and the results are shown in Table 3. The model probability valued $\mathrm{P}<0.0001$ and the corresponding $\mathrm{F}$ value is 8.63 , which shows that the equation model is extremely significant; The $P$ value of the lack-of-fit item is $0.2909>0.05$, indicating that the lack-of-fit item is not significant relative to the pure error. It was proved that the quadratic regression model could predict the effects of soaking time, fermentation temperature, fermentation time and inoculation amount on natto kinase activity and the analysis results were accurate and reliable. The model $\mathrm{B}$ is extremely significant $(\mathrm{P}<0.01)$ and $\mathrm{D}$ is significant $(\mathrm{P}<0.05)$, indicating that the fermentation temperature and inoculum has a significant effect on the activity of natto kinase in the fermentation of natto and the effect of fermentation temperature on enzyme activity was greater than that of inoculation amount. The A2, B2, $\mathrm{C} 2$ and D2 in the table are extremely significant, indicating that the four factors have an impact on the enzyme activity in fermentation, but there is no simple linear relationship of the research level; AC significantly indicated that there was an interaction between soaking time and fermentation time, which both affected the enzyme activity, while The $\mathrm{P}$ values of $\mathrm{AD}, \mathrm{BC}, \mathrm{BD}$ and $\mathrm{CD}$ were all greater than 0.05 , indicated that the interaction between the two was not obvious and there was no joint effect of the two on the enzyme activity.

Figure 4 (a-f) is a 3D three-dimensional view of the interaction of two influencing factors and their contour lines. All 3D images are in the shape of a convex curved surface with an opening downward and the highest point appears under the interaction. The contour line reflects whether the interaction between the two factors is 
significant and the condition of the maximum natto kinase activity after the interaction. The flatter the contour and the more obvious the ellipse, the more significant the interaction will be; on the contrary, the more round the contour, the less significant the interaction will be; The smallest ellipse in the contour map represents the conditional value range of the maximum natto kinase activity and the value range of the optimal fermentation process parameter can be determined by the horizontal and vertical corresponding $\mathrm{X} 1$ and $\mathrm{X} 2$ values. For example, as shown in Fig. 4(a), the contour line under the $A B$ interaction is a typical ellipse. Compared with other contour lines, the image under the $\mathrm{AB}$ interaction is flattering, indicating that A (soaking time) and B (fermentation temperature) interaction effect on the activity of natto kinase is more significant. Observing the contour line of the AB interaction, we know that the natto kinase activity of the fermented product can reach the maximum value when the soaking time is $11 \sim 18 \mathrm{~h}$ and the fermentation temperature is within the range of $35 \sim 39^{\circ} \mathrm{C}$ and its kinase activity can reach up to $3.06 \mu / \mathrm{mL}$. Observing and comparing the $3 \mathrm{D}$ graphs and contour lines under the interaction of other factors, it can be seen that the soaking time is 14-16 h, the fermentation temperature is $35-38.5^{\circ} \mathrm{C}$, the fermentation time is $28-31 \mathrm{~h}$ and the inoculum is $5-6 \mathrm{~mL}$, the activity of natto kinase was higher.

Based on the regression analysis of the model equation of natto kinase, the optimal level of the variable was estimated. The optimal conditions proposed by the model are soaking time $15.4 \mathrm{~h}$, fermentation temperature $37^{\circ} \mathrm{C}$, fermentation time $30.2 \mathrm{~h}$ and inoculation volume $6.0 \mathrm{~mL}$. Under this combined condition, the predicted natto kinase activity is about $3 \mathrm{u} \cdot \mathrm{mL}-1$. The predicted value was verified and it was found that the activity of natto kinase under this combined condition was $3.08 \mu \cdot \mathrm{mL}^{-1}$ consistent with the predicted model.

\section{The Physical Properties of Natto Before and After Fermentation}

In the process of fermenting to natto, we measured the physical properties of soybeans. These physical properties include hardness, elasticity, cohesion, viscosity, chewiness and recovery. The measurement time period is before and after fermentation. Soybeans under each parameter combination are a batch. We select 10 13 soybeans of uniform size of the same batch and take the average of the results. Compared with the soybeans before fermentation, the hardness, elasticity, viscosity and chewiness of soybeans after fermentation are greatly reduced, while the cohesion and resilience are not changed much (Annex 1). It means that after fermentation, soybeans become softer and easier to chew. The chewiness values to reflect the continuous resistance of the sample of chewing, which is the product of hardness, cohesion and elasticity. Its comprehensive reflection power is strong and can be used as a typical reference value of physical property determination. From the perspective of the chewiness of soybeans after fermentation, the chewiness under the combination of $14 \mathrm{~h}-37^{\circ} \mathrm{C}-38 \mathrm{~h}-6 \mathrm{~mL}$ is the lowest, $84.68 \mathrm{~g}$, indicating the best taste; However, the chewiness under the combination of $6 \mathrm{~h}-37^{\circ} \mathrm{C}-28 \mathrm{~h}-6 \mathrm{~mL}$ is the largest, $137.47 \mathrm{~g}$, the taste is harder and the sensory are poor. For a more accurate analysis, we used Design-expert 11.0 software to separately analyze the chewiness values of soybeans after fermentation and the conclusions drawn are shown in Fig. 5.

Table 3: Anova table of quadratic regression model for natto kinase activity

\begin{tabular}{|c|c|c|c|c|c|c|}
\hline Coding variables & Sum of squares & Degree of freedom & Mean square & F-value & P-value & Significance \\
\hline Model & 14.07 & 14 & 1.00 & 8.63 & $<0.0001$ & significant \\
\hline A-Soking time & 0.2185 & 1 & 0.2185 & 1.88 & 0.1908 & \\
\hline B-Fermentation temperature & 0.0688 & 1 & 0.0688 & 0.5907 & 0.0041 & ** \\
\hline C- Fermentation time & 0.1152 & 1 & 0.1152 & 0.9894 & 0.3357 & \\
\hline D-Amount of inoculation & 0.4272 & 1 & 0.4272 & 3.67 & 0.0446 & * \\
\hline $\mathrm{AB}$ & 0.5063 & 1 & 0.5063 & 4.35 & 0.0545 & \\
\hline $\mathrm{AC}$ & 0.6909 & 1 & 0.6909 & 5.94 & 0.0278 & * \\
\hline $\mathrm{AD}$ & 0.0577 & 1 & 0.0577 & 0.4955 & 0.4923 & \\
\hline $\mathrm{BC}$ & 0.3775 & 1 & 0.3775 & 3.24 & 0.0918 & \\
\hline $\mathrm{BD}$ & 0.4159 & 1 & 0.4159 & 3.57 & 0.0782 & \\
\hline $\mathrm{CD}$ & 0.1528 & 1 & 0.1528 & 1.31 & 0.2698 & \\
\hline $\mathrm{A}^{2}$ & 1.54 & 1 & 1.54 & 13.27 & 0.0024 & $* *$ \\
\hline $\mathrm{B}^{2}$ & 8.86 & 1 & 8.86 & 76.14 & $<0.0001$ & *** \\
\hline $\mathrm{C}^{2}$ & 1.02 & 1 & 1.02 & 8.79 & 0.0097 & $* *$ \\
\hline $\mathrm{D}^{2}$ & 2.80 & 1 & 2.80 & 24.03 & 0.0002 & $* *$ \\
\hline Residual & 1.75 & 15 & 0.1164 & & & \\
\hline Lack of fit & 1.35 & 10 & 0.1349 & 1.70 & 0.2909 & not significant \\
\hline Pure error & 0.3973 & 5 & 0.0795 & & & \\
\hline Sum & 15.81 & 29 & & & & \\
\hline
\end{tabular}

PS: *significant difference, $\mathrm{P}<0.05$; **very significant, $\mathrm{P}<0.01$ 

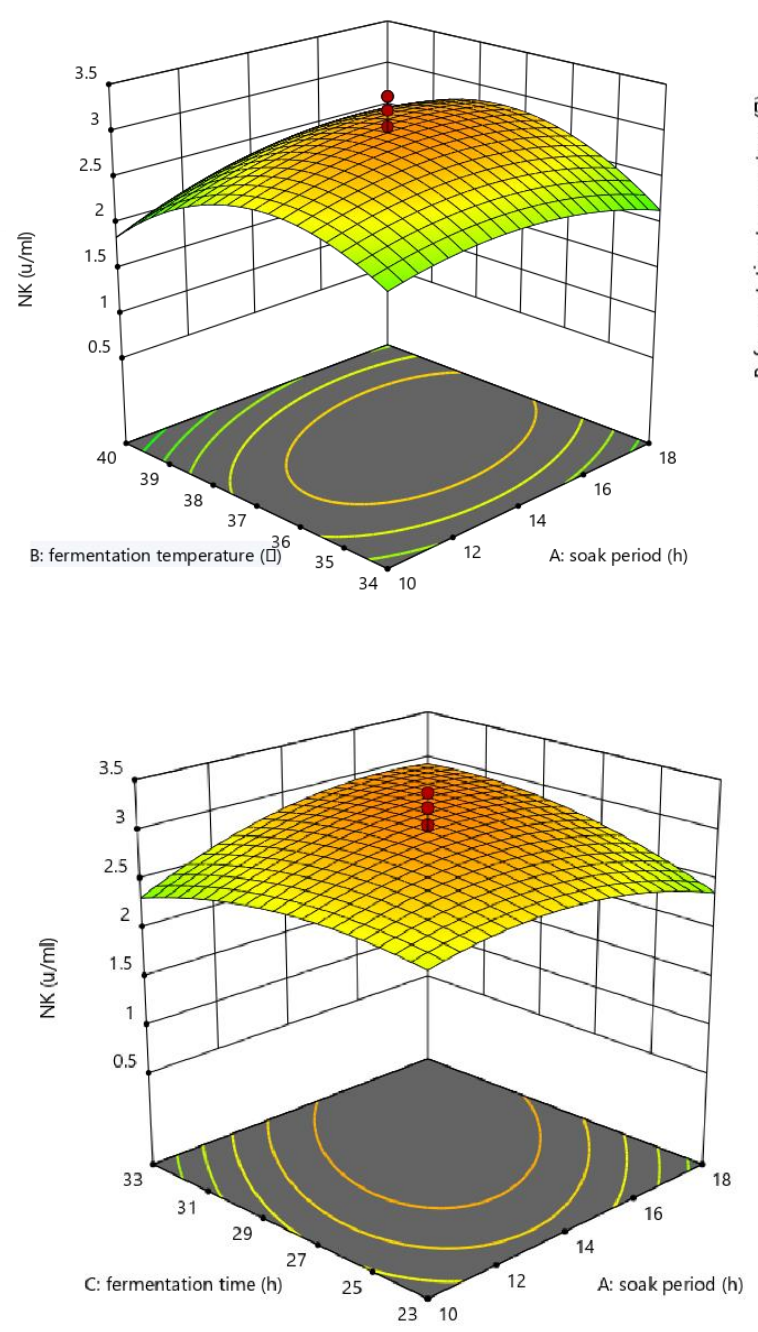

(b)
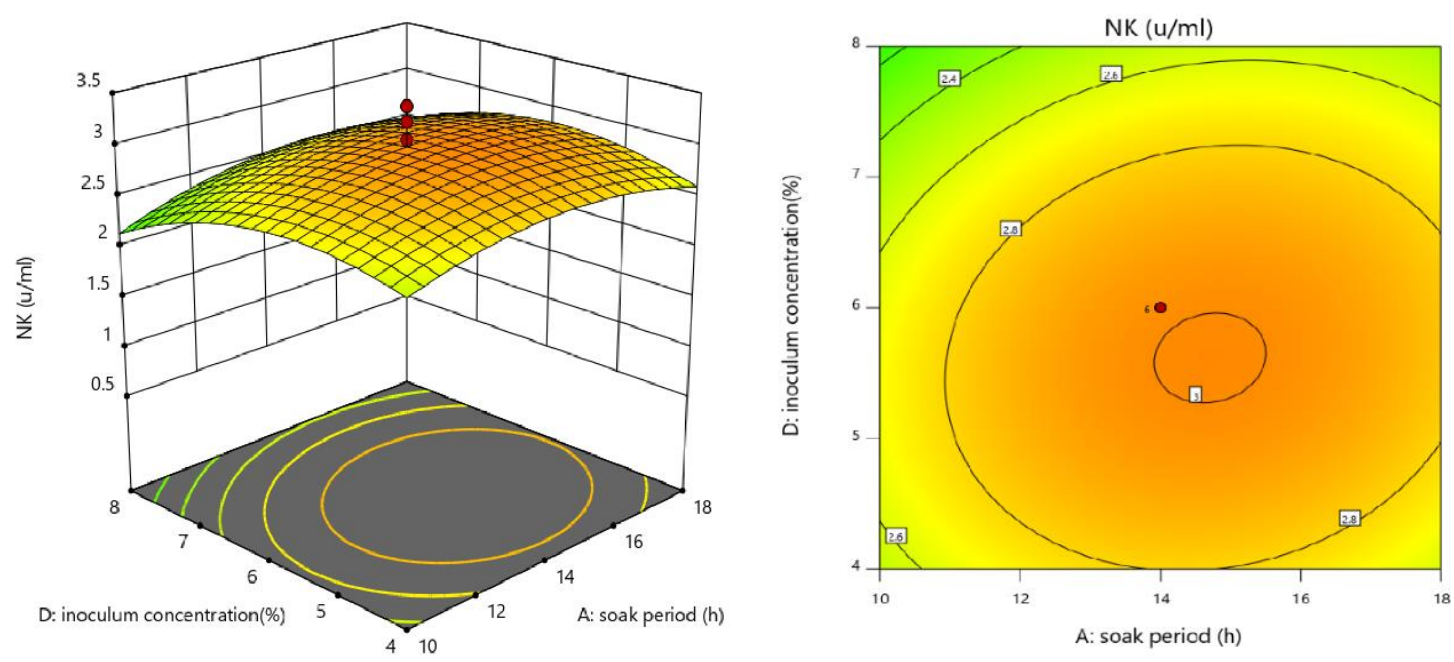

(c)

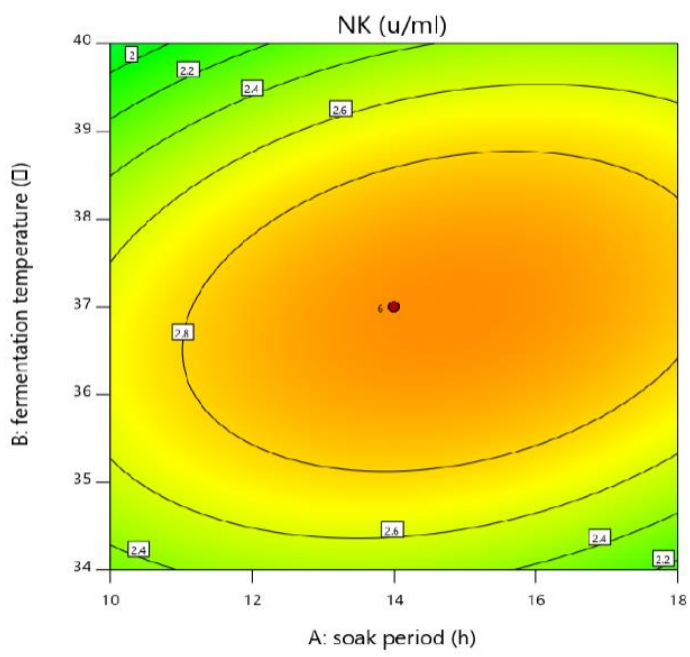

(a)

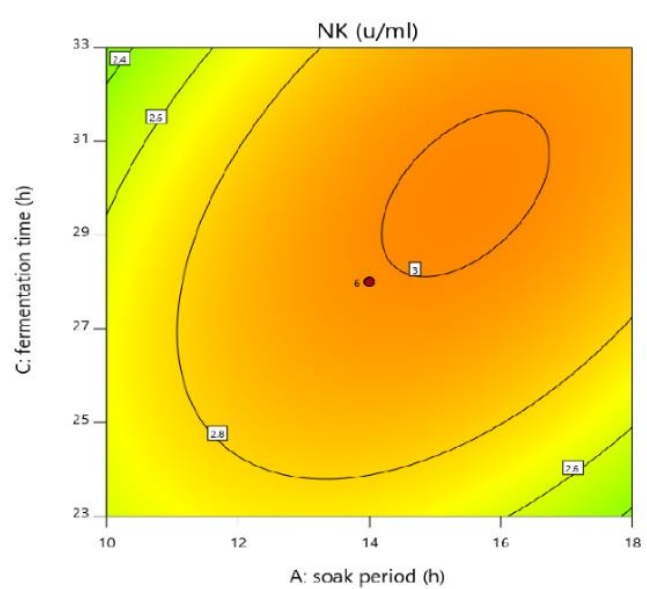



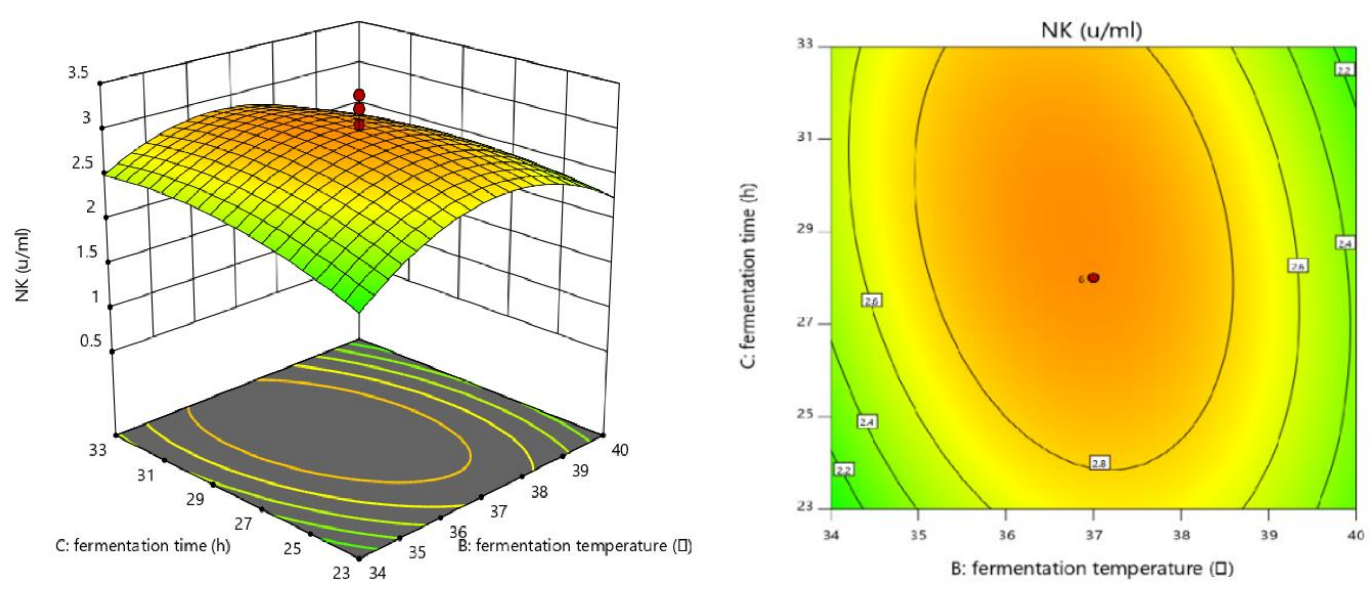

(d)
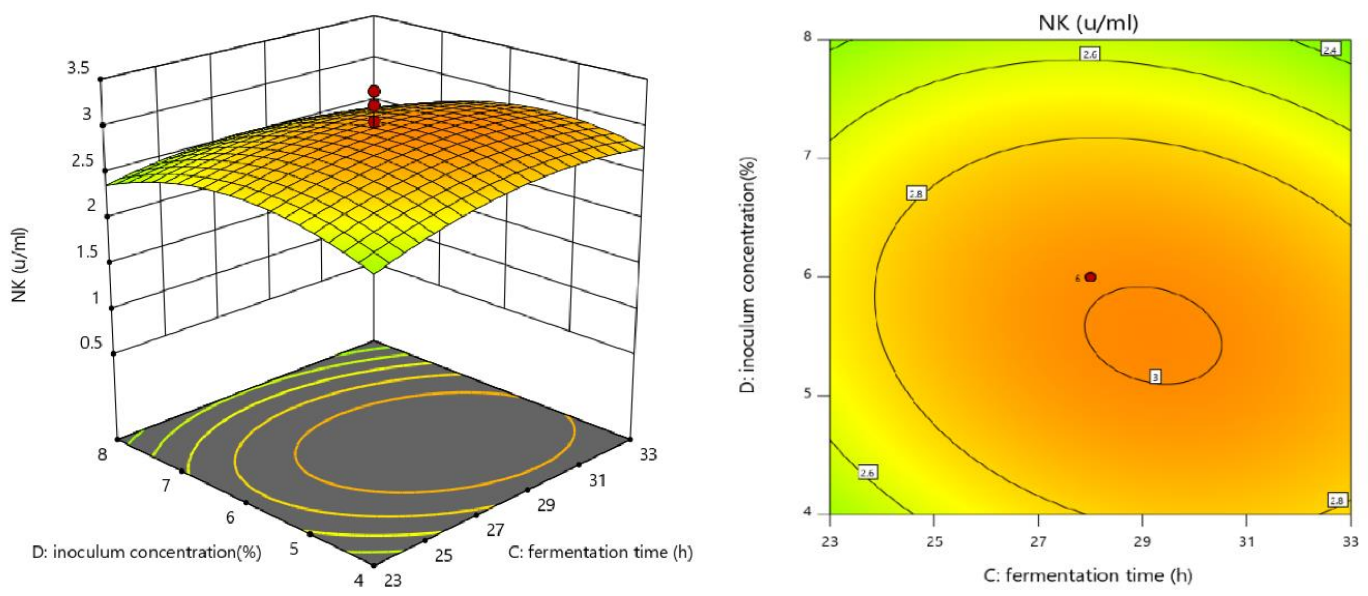

(e)
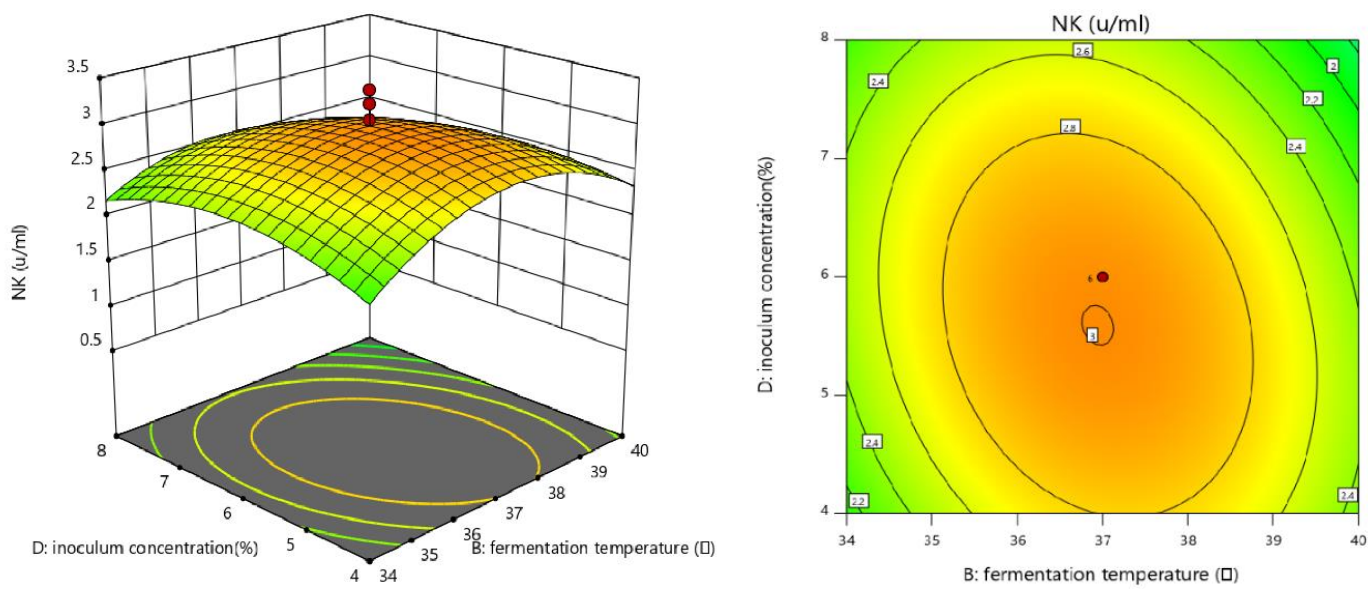

(f)

Fig. 4: Interaction of various factors on natto kinase activity (a) Response surface and contour of the interaction between soaking time and fermentation temperature on natto kinase activity; (b) Response surface and contour of the interaction between soaking time and fermentation time on natto kinase activity; (c) Response surface and contour of the interaction between soaking time and inoculation amount on natto kinase activity; (d) Response surface and contour of the interaction of fermentation temperature and time on natto kinase activity; (e) Response surface and contour of the interaction of fermentation time and inoculation amount on natto kinase activity; (f) Response surface and contour of the interaction between fermentation temperature and inoculation amount on natto kinase activity 

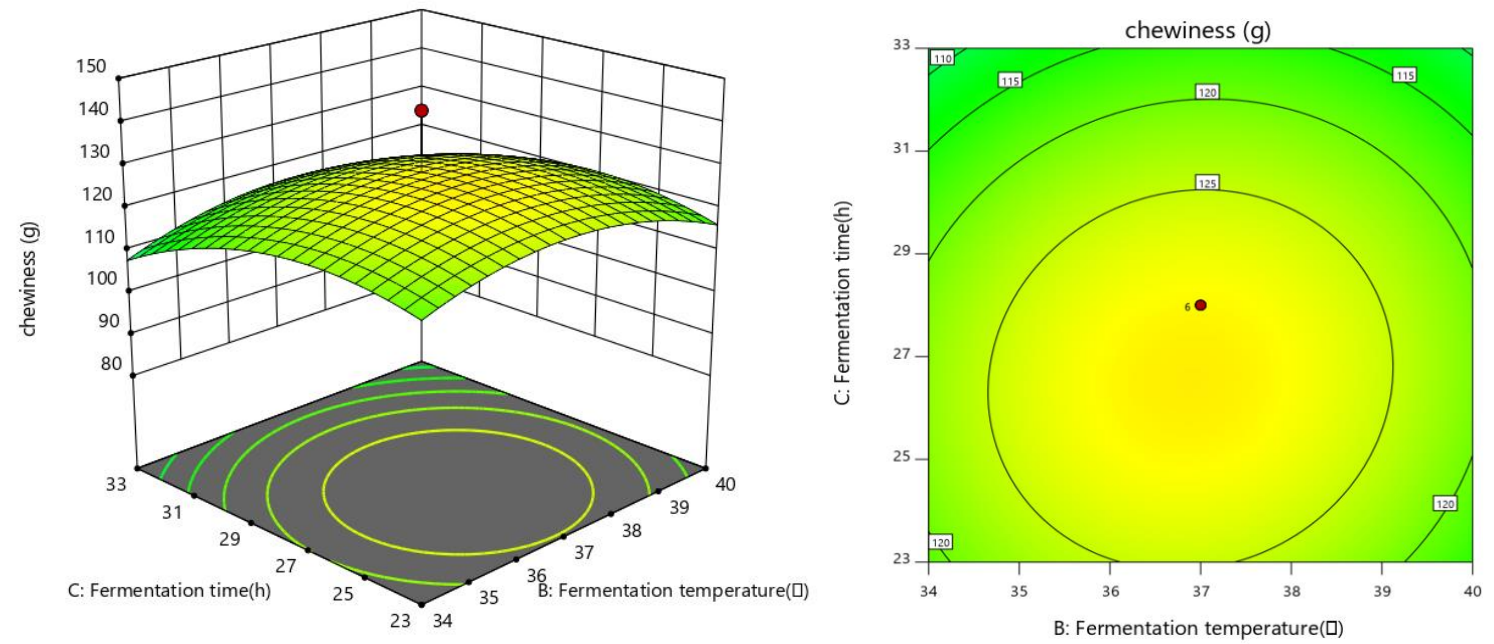

Fig. 5: Interaction of fermentation temperature and fermentation time on chewiness

Through the analysis of variance between the quadratic regression model of the chewiness of soybeans after fermentation, it is concluded that the soaking time has a greater effect on the chewiness of soybeans after fermentation than the fermentation time; There is an interaction between soaking time and fermentation temperature, indicating that the two together affect the chewiness of fermented soybeans; The influence of fermentation temperature on chewiness is not a simple linear relationship. Therefore, proper soaking time and fermentation time can make the fermented natto softer and more delicious.

\section{Conclusion}

This research is based on the traditional natto fermentation process, by optimizing the parameters of the natto fermentation process, to improve the activity of natto kinase. The experiment first determined that Bacillus subtilis natto was the dominant strain of fermenting to natto. Secondly, the influence of soaking and steaming beans on soybean water content was explored and the parameter that affected the activity of natto kinase was further determined to be soaking time rather than cooking time. Through the four-factor fivelevel quadratic rotation orthogonal combination response surface test, 30 sets of fermentation process condition combinations were designed and the natto kinase activity of the fermentation samples was detected by the TAME method. Finally, use Design-expert 11.0 software to analyze the data, analyze the final data through the variance between in the quadratic regression model, the $3 \mathrm{D}$ stereogram under the interaction of each factor and the contour line. The inoculation amount is $6 \mathrm{~mL}$ as the optimal fermentation process condition combination parameter and the natto kinase activity obtained can reach
$3 \mathrm{u} / \mathrm{mL}$. At the same time, a physical property tester was used to measure the samples before and after fermentation, the sensory evaluation was quantified and the chewiness with a strong comprehensive response was represented. It was found that after soaking the beans for $14 \mathrm{~h}$, inoculating $6 \mathrm{~mL}$ and fermenting at $37^{\circ} \mathrm{C}$ for $38 \mathrm{~h}$, the samples were chewed. The lowest sex is $84.68 \mathrm{~g}$. This research can provide a reference to industrial fermentation to produce highly active natto kinase and provide support for the development of related products.

\section{Acknowledgement}

The authors are grateful to the funding support from Shandong Provincial Natural Science Foundation, China (ZR2019BC099 and ZR2020MC201). National Natural Science Foundation of China (31972851 and 31670064). Taishan Industrial Experts Program (tscy20160101). China Post-doctoral Science Foundation (2019M650167 and 2019M662362). Chongqing technological innovation and application development key projects (cstc2019jscx-gksbX0113).

\section{Author's Contributions}

Xiaojie Ren: Participated in the whole experiment process and also contributed to the interpretation of the results.

Chao Huang, Heng Ban, Baoyue Liu and Yu Du: Contributed to the interpretation of the results and manuscript preparation.

Yuanda Song and Xinhe Zhao: Contributed to the guidance of experiment design, ameliorated the manuscript and provided funding support. 


\section{Ethics}

This article is original and contains unpublished material. The corresponding author confirms that all of the other authors have read and approved the manuscript and no ethical issues involved.

\section{References}

CangXue, L., Xiao Yu, L., \& Wen, Z. (2019). Optimization of fermentation process of chickpea by response surface methodology. China Condiment, 44(2), 51-55.

https://www.cabdirect.org/globalhealth/abstract/201 93212255

Hailin, G. (2014). Research progress of natto kinase for both medicine and food. Biotechnology world, 2014(02), 88-89. doi.org/10.1002/rwm3.20139

Iwai, K., Nakaya, N., Kawasaki, Y., \& Matsue, H. (2002). Antioxidative functions of natto, a kind of fermented soybeans: Effect on LDL oxidation and lipid metabolism in cholesterol-fed rats. Journal of Agricultural and Food Chemistry, 50(12), 3597-3601. doi.org/10.1021/jf0117199

Jensen, G. S., Lenninger, M., Ero, M. P., \& Benson, K. F. (2016). Consumption of natto kinase is associated with reduced blood pressure and von Willebrand factor, a cardiovascular risk marker: Results from a randomized, double-blind, placebo-controlled, multicenter North American clinical trial. Integrated blood pressure control, 9, 95. doi.org/10.2147/IBPC.S99553

Ju, S., Cao, Z., Wong, C., Liu, Y., Foda, M. F., Zhang, Z., \& Li, J. (2019). Isolation and optimal fermentation condition of the Bacillus subtilis Subsp. natto strain WTC016 for nattokinase production. Fermentation, 5(4), 92. doi.org/10.3390/fermentation5040092

Junfeng, Z., Shuai, L., \& Pengyan, Z. (2020). Thrombolytic activity of nattokinase produced by Bacillus subtilis natto LNUB236.Food Science, 2020, (13), 148-159. doi.org/10.7506/spkx 1002-6630-20190528-339

Pengyu, Y., Fanbo, M., Daomei, H., Xiuyan, Z., \& Mao, L. (2021). Correlation between texture0analyzer parameters and physical properties measurement of peanut. Modern Food Science and Technology, 2021, 37(07), 294-301. doi.org/10.13982/j.mfst.1673-9078.2021.7.1105
Shih, M. C., Yang, K. T. U., Su, S. Y., \& Tsai, M. L. (2013). Optimization process of roasted broken black soybean natto using response surface methodology. Journal of Food Processing and Preservation, 37(5), 474-482. doi.org/10.1111/j.1745-4549.2011.00669.x

Takagaki, S., Suzuki, M., Suzuki, E., \& Hasumi, K. (2020). Unsaturated fatty acids enhance the fibrinolytic activity of subtilisin NAT (nattokinase). Journal of food biochemistry, 44(8), e13326. doi.org/10.1111/jfbc.13326

Tamura, Y., \& Takenaka, T. (1999). Antioxidative activity of water soluble extracts from okara fermented with Bacillus natto and Rizopus oligosporus. Nippon Shokuhin Kagaku Kogaku Kaishi, 46(9), 561-569. doi.org/10.3136/nskkk.46.561

Vianney, Y. M., Tjoa, S. E. E., Aditama, R., \& Putra, S. E. D. (2019). Designing a less immunogenic nattokinase from Bacillus subtilis subsp. natto: A computational mutagenesis. Journal of molecular modeling, 25(11), 1-12. doi.org/10.1007/s00894-019-4225-y

Wang, Y., Wang, J., Zhang, X., Tong, Y., \& Yang, R. (2021). Genomic and transcriptomic analysis of Bacillus subtilis JNFE1126 with higher nattokinase production through ultraviolet combined $60 \mathrm{Co}-\gamma$ ray mutagenesis. LWT, 147, 111652. doi.org/10.1016/j.lwt.2021.111652

Xiaodan, S., Jialin, W., Jun, M. (2021). Research status and prospect of natto. The Food Industry, 2021,42(07), 227-230.

Yinxin, X., Zongning, Y., Chao, Y., \& Yanfa, M. (2006). Method for determination of nattokinase activity [J]. Pharmaceutical Biotechnology, 2006, 66-69. doi.org/10.3969/j.issn.1674-229X.2000.06.005

Yongjun, W., Dongxu, J., Jiafu, W., \& Shounian, Z. (2010). Preliminary study on primary fermentation conditions of Bacillus of lobster sauce and its enzyme activity assay. Science and Technology of Food Industry, 2010, 31(09), 191-194. doi.org/10.13386/j.issn1002-0306.2010.09.074

Zexin, G., Laping, H., Yabin, L., Bin, G., Cui, Q., \& Hanyu, L. (2017). Research progress and prospect of nattokinase. China Brewing, 2017, 17-21. doi.org/16736/j.cnki.cn41-1434/ts.2018.16.012 